\title{
What is the place of lumbar sympathectomy?
}

\section{S. M. LINDENAUER AND J. L. CRONENWETT}

Department of Surgery, Lniversity of Michigan, Ann Arbor, Michigan 48109. USA

\author{
Although sympathetic innervation of the extremities has been studied \\ voluminously, the clinical efficacy of sympathectomy remains \\ controversial. This review will be limited to a consideration of the \\ appropriate role of lumbar sympathectomy for primary treatment of \\ intermittent claudication and skin ischaemia manifested by rest pain \\ or superficial tissue loss.
}

Skeletal muscle is diffusely innervated by sympathetic nerves but the effect of sympathetic discharge is limited to resting muscle blood flow. Rapidly responding muscle fibres, which predominate in human skeletal muscle, receive an average resting blood flow of $2-5 \mathrm{ml} 100 \mathrm{~g}^{-1} \mathrm{~min}^{-1}$, depending on the level of sympathetic vasoconstrictor activity, which is regulated primarily by the baroreceptor response to systemic blood pressure. Although maximal sympathetic discharge (6-10 impulses/s) will lower resting muscle blood flow to 15 per cent of its normal value, elimination of all vasoconstrictor activity only increases this flow to approximately $6-9 \mathrm{ml} 100 \mathrm{~g}^{-1} \mathrm{~min}^{-1}$ (1). This contrasts with flow to exercising muscle of $50-75 \mathrm{ml} 100 \mathrm{~g}^{-1} \mathrm{~min}^{-1}$, which is the same in normal and sympathectomized limbs. Thus, afferent arterioles in skeletal muscle have a high intrinsic myogenic tone which compensates for sympathetic ablation. During exercise, locally produced metabolic substances cause maximal vasodilation, despite sympathetic discharge. Patients who experience intermittent claudication already have maximal muscle arteriolar vasodilation, due to ischaemia. The extent of this ischaemia depends on the adequacy of collateral vessels.

While sympathectomy in claudicants cannot increase exercise hyperaemia by influencing the already dilated muscle capillary bed, it has been proposed that sympathectomy might dilate collateral arteries. If this occurred in extremities where blood flow was limited by small collateral channels, inflow to a maximally dilated peripheral vascular bed might be increased. There is little evidence, however, that sympathetic nerves exert any vasoconstrictive influence on large muscular arteries. Although collateral arteries clearly enlarge after a period of time following major arterial occlusion, this appears to result from a gradual change in intrinsic myogenic tone, rather than the elimination of sympathetic vasoconstrictive activity. Sympathectomy results in a transient increase in collateral blood flow in ischaemic extremities, but we believe that this increase is a passive one, resulting from decreased distal resistance due to maximal dilatation of arteriovenous anastomoses (AVAs). In ischaemic canine hindlimbs following femoral artery ligation, we have shown that vasodilation following sympathectomy results from the opening of AVAs, rather than skin or muscle arterioles, despite a measurable oxygen debt in the extremity (2). As previously observed, transiently increased total extremity blood flow in ischaemic extremities after sympathectomy has been attributed to active improvement of collateral flow (3). This increase in total extremity flow, however, is readily explained by dilatation of distal AVAs, with passive increase in collateral flow. This occurs because AVAs are not already dilated in ischaemic extremities, in contrast to afferent arterioles. In the unlikely event that large muscular collateral arteries are even transiently responsive to ablation of sympathetic vasoconstriction, it is doubtful that the standard L2-L4 sympathectomy is responsible, since most significant collaterals are proximal to the area of denervation.

Direct measurement of muscle capillary blood flow after sympathectomy has also been accomplished, using isotope clearance, microsphere uptake and mass spectrometry (4-7). Such studies have repeatedly failed to document a significant change in either resting or hyperaemic muscle blood flow.
These data notwithstanding, numerous clinical reports contend that patients with intermittent claudication demonstrate improved exercise tolerance following sympathectomy. Unfortunately, these studies have been uncontrolled. Objective experiments by Jacobson and Bush (8) showed no demonstrable effect of sympathectomy on exercise tolerance or on the rate of collateral development in dogs following sympathectomy. In a carefully controlled human trial, Fyfe and Quin (9) showed that only 25 per cent of patients undergoing phenol sympathectomy claimed subjective improvement, while 45 per cent of control patients claimed such improvement after 3 months. Furthermore, objective treadmill exercise tolerance and distal extremity blood pressure showed no significant difference between control and sympathectomy groups (9).

The variable natural history of atherosclerotic occlusive disease, the known placebo effect of any therapeutic intervention and the influence of other variables such as cigarette smoking and exercise make the subjective evaluation of intermittent claudication impossible. Unfortunately, an abundance of uncontrolled reports have led to subjective enthusiasm regarding the usefulness of sympathectomy to treat intermittent claudication. We do not believe that there are physiological or objective clinical data to support such treatment.

Resting skin blood flow, as opposed to muscle blood flow, is determined primarily by the demands of thermal homeostasis, not by local metabolic requirements. Thus, skin blood flow is regulated primarily by sympathetic control of the amount of blood flowing through cutaneous AVAs. These AVAs are densely innervated muscular channels capable of dilating to $50 \mu \mathrm{m}$ diameter. Their presence and function are well documented anatomically in acral body parts. AVAs are capable of rapid phasic responses and, because of their large potential diameter, can accommodate tremendous increases in blood flow with a marked reduction in peripheral resistance. In contrast to afferent arterioles in the nutritive capillary system, AVAs have little or no intrinsic resting myogenic tone, but are dependent upon sympathetic vasoactivity to control their diameter (1). Furthermore, AVAs do not respond to ischaemia with the customary vasodilation seen in muscular or skin capillary beds. In fact, using microspheres to measure AVA and capillary flow in dogs directly, we have shown that, during reactive hyperaemia, flow through AVAs remains constant, despite large increases in muscle and skin capillary flow (10) Resting skin blood flow is significantly higher per gramme of tissue than resting muscle blood flow due to its temperature regulating function. Increased core (hypothalamic) temperature has been demonstrated to increase skin AVA flow significantly, thus enhancing heat dissipation, while reduced core temperature decreases AVA flow, conserving heat (11). Both of these functions are mediated by changes in sympathetic vasoconstrictor tone. Effects of local extremity heating and cooling, however, are predominantly mediated by changes in capillary, rather than AVA, flow. These effects are independent of sympathetic control. Delius et al. (12) have measured skin sympathetic nerve impulses in resting awake humans and have shown that other stimuli besides thermal changes can modulate sympathetic output (12). Mental and emotional stress, cigarette smoking and hyperventilation regularly increase activity in these nerves. Conversely, body position, Valsalva manoeuvre, 
blood pressure and muscle work have no effect on skin sympathetic discharge, while they significantly change resting muscle sympathetic activity.

Skin capillaries, in contrast to AVAs, exhibit a relatively high baseline myogenic tone which is influenced little by sympathetic denervation in animal experiments (1). Thus, sympathectomy would be expected to have little effect upon skin capillary blood flow. AVAs, however, should significantly dilate after sympathectomy, resulting in increased skin temperature and increased total extremity blood flow as measured by plethysmography or proximal large vessel flowmetry. By directly measuring the blood flow partitioned through AVAs versus capillaries using radioactive microspheres in both normal and ischaemic canine hindlimbs, we have shown this to be the case (2). Blood flow through AVAs following acute lumbar sympathectomy was increased seven to eightfold in canine hindlimbs and entirely accounted for the increase in total extremity blood flow. As expected, this was associated with a significantly increased paw skin temperature.

Despite these experimental observations, there exist many clinical reports which suggest that approximately 50-60 per cent of patients undergoing lumbar sympathectomy for treatment of skin ischaemia (rest pain or superficial ulceration) experience significant improvement and in some cases avoid amputation. Unfortunately, increased skin temperature is often erroneously cited as evidence of a beneficial effect of sympathectomy. Although reassuring when contrasted to the previously cold ischaemic extremity before operation, increased skin temperature is not due to improved capillary blood flow, but rather is due to increased non-nutritive AVA flow. Similarly, increased total extremity blood flow measured by plethysmography or large vessel flowmetry or inferred from an improved ankle or toe blood pressure cannot be attributed to improved nutritive blood flow, since the marked reduction in peripheral resistance resulting from dilated AVAs causes a tótal flow increase.

Support for the concept that improved nutritive skin blood flow is due to lumbar sympathectomy, therefore, must rely upon direct measurement of skin capillary blood flow. Unfortunately, our ability to measure small changes in skin blood flow over a non-homogeneous body surface, subject to substantial changes related to environmental temperature, is not precise and has resulted in conflicting data. Using tissue clearance of subcuticularly injected radio-isotopes, some investigators have reported improved skin capillary blood flow following sympathectomy (13), while others have reported no change in capillary skin flow (14). Polarographic measurements of skin oxygen tension following sympathetic denervation showed a decrease in skin oxygen tension, despite the presence of cutaneous hyperaemia attributed to AVAs (15). Using mass spectrometry, tissue oxygen tension was unchanged in canine hindlimb subcutaneous tissue and muscle following sympathectomy (7). We have recently measured nutritive skin blood flow in canine hindlimbs after sympathectomy by using tissue uptake of radioactive labelled $15 \mu \mathrm{m}$ diameter microspheres in both awake and anaesthetized animals (6). These data demonstrate no significant change in skin blood flow after sympathectomy in dogs, despite increases in total extremity blood flow due to increased AVA flow.

Despite these attempts to measure skin blood flow after sympathectomy, the final burden of proof regarding the value of sympathectomy in patients with skin ischaemia should rest upon controlled clinical trials. Although sympathectomy has been performed for this indication for over 50 years, such controlled objective clinical studies are non-existent. Based upon physiological principles and skin blood flow measurement, there are no convincing data that sympathectomy would be beneficial in this clinical circumstance. Even if one postulates that increased sympathetic vasoconstrictor activity due to stress or cigarette smoking might influence skin capillaries in addition to AVAs, it is doubtful that such vasoconstriction could exceed the vasodilating effect of local metabolic substances already present in ischaemic skin.

Owing to the reported variability in clinical response to lumbar sympathectomy, attempts were made to predict which patients would respond favourably. Such predictions have generally been based upon tests which document the presence of intact sympathetic nerves by inducing transient sympathetic blockade and measuring skin temperature, skin impedance or plethysmographically determined total extremity blood flow. Although these techniques can define intact sympathetic innervation of extremities (as does the simpler measurement of the toe pulse volume follow deep inspiration), they measure both AVA and capillary flow. Confirming the ability to eliminate resting sympathetic tone by these techniques does not alter the underlying fact that sympathetic denervation does not significantly augment nutritive capillary blood flow. Based upon such 'predictive' studies, it is generally suggested that patients who will respond favourably to sympathectomy are those with ankle systolic pressures greater than $30 \mathrm{mmHg}$ (16). Clearly, these are the very patients most likely to improve if non-operative care is administered to their extremity.

Lumbar sympathectomy is not an innocuous clinical procedure. It is associated with a postoperative mortality reported in 1976 to be 6.5 per cent (17). It adds increased hospitalization, morbidity and cost to what might otherwise be a judicious amputation and rehabilitation. There is no sound scientific basis to support the use of lumbar sympathectomy for the treatment of intermittent claudication. Its use for the treatment of skin ischaemia has not been proved by appropriately controlled clinical trials. Unfortunately, this is as true today as it was in 1967, when Szilagy stated: 'the evidence must be brought in by those who make the claim for the effectiveness of the procedure rather than by those who will not accept the claim until its proof is at hand' (18).

\section{References}

1. Folkow B. and Neil E.: Circulation. New York: Oxford University Press, 1971.

2. Cronenwett J. L. and Lindenauer S. M.: Hemodynamic effects of sympathectomy in ischemic canine limbs. Surgery 1980: 87: 417-24

3. Dornhorst A. C. and Sharpey-Schafer E. P.: Collateral resistance in limbs with arterial obstruction: spontaneous changes and effects of sympathectomy. Clin. Sci. 1951; 10: 371-81.

4. Hirai M., Kawai S. and Shionoya S.: Effect of lumbar sympathectomy on muscle circulation in dogs and patients. Nagoya J. Med. Sci. 1975; 37: $71-7$.

5. Hoffmann D. C. and Jepson R. P.: Muscle blood flow and sympathectomy. Surg. Gynecol. Obstet. 1968; 127: 12-16.

6. Cronenwett J. L. and Lindenauer S. M.: Effect of sympathetic innervation on canine muscle and skin blood flow. (In preparation.)

7. Perry M. and Horton J.: Muscle and subcutaneous oxygen tension. Arch. Surg. 1978; 113: 176-8.

8. Jacobson J. H. and Bush H. S.: Effect of bilateral lumbar sympathectomy on experimentally produced intermittent claudication. Surgery 1963; 54: 617-19.

9. Fyfe T. and Quin R. O.: Phenol sympathectomy in the treatmen of intermittent claudication: a controlled clinical trial. Br.J. Surg. 1975; 62: 68-71.

10. Cronenwett J. L and Lindenauer S. M. Direct measurement of arteriovenous anastomotic blood flow after lumbar sympathectomy. Surgery 1977; 82: 82-9.

11. Hales J. R. S., Fawcett A. A., Bennett J. W. et al.: Therma control of blood flow through capillaries and arteriovenous anastomoses in skin of sheep. Pfuegers Arch 1978; 378: 55-63.

12. Delius W., Hagbarth K-E., Hongell A. et al.: Manoeuvres affecting sympathetic outflow in human skin nerves. Acta Physiol Scand. 1972; 84: 177-86.

13. Moore W. S. and Hall A. D.: Effects of lumbar sympathectomy on skin capillary blood fow in arterial occlusive disease. $J$. Surg. Res. 1973; 14: $151-7$

14. Carr M. J. T., Crooks J. A., Griffiths P. A. et al.: Capillary blood flow in ischemic limbs before and after surgery assessed by subcuticular injection of Xenon 133. Am. J. Surg. 1977; 133 $584-6$.

15. Davis M. T. and Greene N. M. : Polarographic studies of skin oxygen tension following sympathetic denervation. J. Appl. Physiol. 1959; 14: $941-5$.

16. Walker P. M. and Johnston M. D.: Predicting the success of a sympathectomy: a prospective study using discriminant function and multiple regression analysis. Surgery 1980; 87: 216-21.

17. Kim G. E., Ibrahim I. M. and Imparato A. M.: Lumbar sympathectomy in end stage arterial occlusive disease. Ann. Surg. 1976; 183: $157-60$

18. Szilagy D. E., Smith R. F., Scerpella J. R. et al.: Lumbar sympathectomy: current role in the treatment of arteriosclerotic occlusive disease. Arch. Surg. 1967; 95: 753-61. 